\title{
Circuit
}

Musiques contemporaines

\section{Empreintes Digitales, une étiquette qui laisse des traces}

\section{Réjean Beaucage}

Volume 12, numéro 3, 2002

La route de soi

URI : https://id.erudit.org/iderudit/402005ar

DOI : https://doi.org/10.7202/402005ar

Aller au sommaire du numéro

Éditeur(s)

Les Presses de l'Université de Montréal

ISSN

1183-1693 (imprimé)

1488-9692 (numérique)

Découvrir la revue

Citer cet article

Beaucage, R. (2002). Empreintes Digitales, une étiquette qui laisse des traces. Circuit, 12(3), 63-70. https://doi.org/10.7202/402005ar

\section{Résumé de l'article}

Recension de la production discographique de l'étiquette Empreintes Digitales pour 2001, une année consacrée à la mise à jour du versant québécois du catalogue avec des nouveautés d’Yves Daoust, Francis Dhomont, Gilles Gobeil et Robert Normandeau, une édition des oeuvres électroacoustiques de la regrettée Micheline Coulombe Saint-Marcoux et un premier disque pour Yves Beaupré. d'utilisation que vous pouvez consulter en ligne.

https://apropos.erudit.org/fr/usagers/politique-dutilisation/ 


\title{
Empreintes Digitales, une étiquette qui laisse des traces
}

\author{
Réjean Beaucage
}

La maison de disques Empreintes Digitales célèbre cette année son douzième anniversaire. Au fil des ans, la petite étiquette montréalaise a accouché d'une montagne, incontournable Everest des amateurs de musique électroacoustique d'ici el d'ailleurs. L'année 2001 a été consacrée à une mise à jour du versant québécois du catalogue avec des nouveautés d'Yves Daoust', Francis Dhomont ${ }^{2}$, Gilles Gobeil ${ }^{3}$ et Robert Normandeau ${ }^{4}$. On a aussi eu la très bonne idée d'éditer les œuvres électroacoustiques de la regrettée Micheline Coulombe Saint-Marcoux ${ }^{5}$ et on nous présente un nouveau venu en la personne d'Yves Beaupré ${ }^{\circ}$, compositeur et facteur de clavecin. Bref, une importante mise à jour qui permet de brosser, en un coup d'œil, un portrait des différentes générations d'électroacousticiens.

Le disque Impulsion regroupe l'ensemble des pièces pour bande seule de Micheline Coulombe Saint-Marcoux, à l'exception de deux œuvres antérieures qui n'ont pu être retracées. On ne peut que féliciter l'étiquette Empreintes Digitales pour cette initiative qui évitera que le travail de SaintMarcoux ne sombre dans l'oubli, comme tant d'autres créations musicales d'ici (et d'ailleurs). Quatre des cinq pièces reproduites datent du début des années soixante-dix. Cela s'entend ef nous réjouit, parce qu'elles nous ramènent à un moment de l'histoire de la musique électroacoustique, juste après les premiers balbutiements de celle-ci, durant lequel, bien que toutes les portes aient été ouvertes, on pouvait encore croire à ce que le meilleur reste à venir. Une époque où la musique était encore assez concrète pour tenir dans la main, être coupée, retoumée, puis collée.

Micheline Coulombe Saint-Marcoux faisait partie du groupe historiquement marginal des "antispécialistes", pour qui la recherche sonore ne connaissait pas de frontière. Synthèse électronique et montage de bande magnétique avec variation de vitesse font bon ménage ef offrent à la composition une palette de sons d'une grande richesse. C'est lannis Xenakis, lors d'un passage au Conservatoire de musique du Québec à Montréal, à l'invitation de Gilles Tremblay, qui aurait suggéré à Micheline Coulombe Saint-Marcoux d'aller dépenser son Prix d'Europe au sein du Groupe de recherches musicales à Paris (GRM) en 1968. La fréquen-
1. Yves Daoust, Bruits, Empreintes Digitales/Diffusion i Média, 2001 (IMED $0 \mid 561$.

2. Francis Dhomont, Cycle du son, Empreintes Digitales/Diffusion i Média, 2001 (IMED 0158).

3. Gilles Gobeil, ... dans le silence de la nuit..., Empreintes Digitales/Diffusion i Média, 2001 (IMED 0 155).

4. Robert Normandeau, Clair de terre, Empreintes Digitales/Diffusion i Média, 2001 (IMED 0157).

5. Micheline Coulombe Saint-Marcoux, Impulsion, Empreintes Digitales/Diffusion i Média, 2001 (IMED 0159 ).

○. Yves Beaupré, Humeur de facteur, Empreintes Digitales/Diffusion i Média, 2001 (IMED 0 160 ). 
tation des François Bayle, Henri Chiarucci ef Guy Reibel au GRM, de même que les enseignements du pionnier Pierre Schaeffer au Conservatoire national supérieur de musique de Paris, ont certes été des éléments déterminants du travail compositionnel de Saint-Marcoux. Les trois premières pièces du disque, réalisées à Paris en 1970 et 1971, portent indubitablement la marque du GRM tout en témoignant d'une grande originalité.

Basée sur des textes des poètes Noël Audet et Gilles Marsolais, Arksalalartôq évoque les jeux vocaux des femmes inuits, joutes verbales absurdes qui se terminent souvent par les éclats de rire des participantes. Le travail sur la voix rappelle celui du Pierre Henry de Granulométrie et le traitement de sons électroniques salue le Stockhausen de Gesang der Jünlinge. L'étude Contrastances se rapproche, comme l'indique son titre, des matériaux sonores en opposition, tant en ce qui concerne la vitesse que la hauteur ou la texture. Élaborée, d'après le livret, "dans un studio privé à Paris», le matériau de base y est moins varié que dans les deux autres pièces de la même époque, réalisées au GRM. Cependant, le résultał demeure étonnant et la luxuriance qui s'en dégage est certes à mettre au crédit de la compositrice. La dernière des œuvres parisiennes, Moustières, est une belle démonstration de la maîtrise des techniques acquises durant la composition des œuvres précédentes et elle "coule " dans l'oreille comme une construction sans faille. La pièce Zones a quant à été réalisée à l'Université Simon Fraser de Vancouver entre 1971 et 1972, principalement à partir de sons produits par différents instruments à clavier (piano, orgue, clavecin ou claviers électroniques). L'œuvre est constituée de quatre différentes "zones» qui se distinguent par les techniques de transformation utilisées sur les sons de base, "[l]'idée [étant] d'arriver à la fusion des qualités sonores en manipulant de la même façon les sons d'origine ${ }^{7}$ ". Le geste y est plus long que dans les pièces précédentes et laisse présager du style que développera Saint-Marcoux dans ses compositions instrumentales subséquentes. Le disque s'achève avec la pièce la plus longue et la plus récente, Constellation I, qui date de 1981. Elle aussi est construite sur le principe de l'équilibre des contrastes, le plan de l'œuvre s'articulant en une juxtaposition de masses texturales assemblées par des charnières de silence. On y traverse encore des "zones", chacune étant en quelque sorte un microcosme du plan général. On pourrait mettre l'œuvre en boucle et ne plus jamais sortir de ce paysage.

Il est intéressant de trouver dans la notice biographique du compositeur Yves Daoust, telle que reproduite dans le livret de son disque Bruits, une liste des influences qui marquèrent le travail de Daoust. On y découvre les «trames sonores de films, bien sûr», mais aussi les compositeurs Cage, Xenakis, Kagel, Ferrari, Savouret, Stockhausen (ici, il précise Hymnen), Beethoven et Schumann, et elle se termine par le nom du peintre surréaliste René Magritte. Glisser le nom de ce peintre du détournement à la suite de ceux de compositeurs parmi les plus inventifs n'est certainement
7. Les notes du livret sont de Micheline Coulombe Saint-Marcoux. 
pas innocent de la part du compositeur. Daoust travaille fréquemment à partir de sons réalistes, ou "naturels", que l'auditeur peut identifier immédiatement, comme l'observateur peu identifier aisément tous les éléments entrant dans la composition d'une toile de Magritte. C'est leur agencement, ou leur traitement, qui peut être déstabilisant, un peu «comme la rencontre fortuite sur une lable de dissection d'une machine à coudre et d'un parapluie ${ }^{8}$. Daoust tient à nous ancrer dans la réalité afin de mieux nous amener ailleurs. On serait tenté de parler de musique surréaliste mais aussi de cinéma sonore. D'autant plus qu'il ne faut pas oublier que le compositeur fut quelque temps concepteur sonore à l'ONF et qu'il vécut ses premières expériences électroacoustiques à 16 ans, en sonorisant le film $8 \mathrm{~mm}$ d'un ami.

Le disque s'ouvre sur Bruits, suite en trois tableaux / Children's Corner», "Nuit " et "Fête" qui utilise abondamment les images sonores citadines les plus convenues, "fêtes de rue, marché, jeux extérieurs, machines, jardins publics [...] clameurs humaines, cris et sifflets ${ }^{9} "$, dans un ballet qui n'a rien du documentaire ef tout du poème... bruitiste. Le "paysage" y est hachuré et le tourbillon sonore finit par s'épaissir en une soupe dense d'où s'arrachent par bribes des morceaux de réalité, comme des éclats de lumière fuyant le magnétisme du trou noir. On notera en effet au passage l'influence avouée de Luc Ferrari.

La pièce Impromptu apparaissait déjà sur le disque précédent qu'a fait paraître Daoust chez Empreintes Digitales, Musiques naïves, en version pour bande seule. L'œuvre a été largement retravaillée dans cette version mixte, pour piano, synthétiseur/échantillonneur et bande. La partie de piano est confiée à Jacques Drouin, et Lorraine Vaillancourt joue la partie de clavier électronique. La pièce a été réalisée "grâce à la complicité de Chopin ", de qui Daoust emprunte et détourne quelques motifs de la Fantaisie-Impromptu en do dièse mineur, op. 66, pour en faire un objet neuf, mais chargé des mêmes tensions et de ce même spleen qui caractérisaient l'époque romantique. Une grande réussite dans un ton que l'on trouve peu souvent en électroacoustique.

La gamme nous ramène à une autre époque. Le compositeur nous laisse ici assister à ses premiers pas dans le monde de la musique électronique. On y observe en quelque sorte le compositeur se surprendre lui-même de ses découvertes et nous les présenter sans prétention. Alors on s'amuse à écouter la pièce comme le témoignage d'un passé que la vitesse exponentielle des développements technologiques tend à nous faire oublier ll'œuvre a été composée en 1981 !). Le disque se termine par... Ouverture, une œuvre de 1989 commandée à Yves Daoust par le Groupe de musique électroacoustique de Bourges pour commémorer le bicentenaire de la Révolution française. Le thème et son traitement appellent la comparaison avec le trésor de la langue, du compositeur/improvisateur René Lussier, qui date de la même année (1989). Ces deux pièces dénotent une grande communauté d'esprit entre les compositeurs. Ils utilisent des dialogues en forme d'entrevue afin d'établir des rapports sonores entre "le vieux pays" et le Québec. Cette similitude d'intention mérite d'être soulignée puisque nous avons ainsi deux œuvres stylistiquement fort différentes sur un même thème.
8. L'un des fameux «Beau comme..." de Lautréamont, extrait des Chants de Maldoror.

9. Extrait de la notice du livret. 
Ouverture est extrêmement narrative el a peut-être davantage les qualités d'une œuvre radiophonique que celles d'une œuvre de concert. Elle clôt le disque avec l'évocation d'une réalité plus tangible, plus "réelle", que dans les pièces précédentes. On ne rêve plus. Le disque est fini.

La musique de Gilles Gobeil est un guet-apens psychoacoustique. Le compositeur attire l'attention de l'auditeur sur des détails infimes, le laisse en quelque sorte pénétrer dans le son pour admirer sa structure, puis il détourne brusquement son attention par une cassure qui propulse l'auditeur sur une autre piste. Le procédé est d'une efficacité remarquable, mais il est probablement trop systématisé. La qualité des textures sonores que tisse Gobeil est ahurissante. La musique qui en découle est concrète à un point tel que le son y apparaît à l'état solide. Plus question ici de référence à la "réalité » puisque le moindre objet sonore est observé au microscope et révèle ainsi des contours insoupçonnés.

Malgré l'éclatante réussite au plan de la richesse sonore, on peut se demander si le compositeur atteint bien les objectifs descriptifs qu'il définit lui-même. Le résultat étant finalement assez abstrait, on peut se demander s'il était bien pertinent de nous les faire connaître. En effet, les "quelques images d'un voyage en Italie ${ }^{10}$ " qui servent de matériau de base à la pièce Derrière la porte la plus éloignée... (1998) évoquent bien davantage un paysage de la planète Mars qu'une "visite guidée de la cathédrale de Torcello" ${ }^{11}$. Quant à la pièce Projet Proust 11995 , 20011, elle n'a sans doute de proustien que les quelques lignes de texte murmurées par le narrateur (Marc Béland). II s'agit bien sûr d'un problème de perception, le compositeur ne portant pas sur son matériau le même regard que l'auditeur. Les images sonores ont ceci de merveilleux qu'elles débrident complètement l'imagination de celui qui les reçoit. Force est de constater que le mode d'emploi offert a ses limites. Estil donc utile de fournir des pistes si, en définitive, elles ne semblent pas avoir été suivies? Dans ce cas, l'auditeur en a-t-il vraiment besoin?

Point de passage (1997) est une libre adaptation du roman The Time Machine de H. G. Wells et Nuit cendre (1995) s'inspire du Voyage au centre de la Terre de Jules Verne. L'auditeur qui l'ignorerait n'en serait pas moins transporté dans un autre temps, un autre lieu.

dans le silence de la nuit... est le deuxième disque de Gilles Gobeil chez Empreintes Digitales et chacune des pièces qu'il contient a reçu au moins une distinction dans une compétition internationale. Ce qui n'a rien d'étonnant, puisque la musique de Gobeil est séduisante. Avec un bon dosage des effets de surprises, la densité des matières qu'il utilise, le compositeur produit des objets particulièrement scintillants, qui ne se contentent d'ailleurs pas d'être bien construits. L'équilibre qui règne dans ses œuvres n'a pas la fragilité de celui du fildefériste, mais plutôt l'assurance des mobiles de Calder.

10. Extrait de la notice du livret.

11. Idid. 
De la même génération que Gilles Gobeil et, pour ainsi dire, de la même école, Robert Normandeau a une approche bien différente de l'électroacoustique. À la fois plus "musicales", au sens où l'utilisation des codes de la musique instrumentale y est plus perceptible, et plus théoriques, les pièces de Normandeau possèdent une identité précise tant en ce qui concerne les sources utilisées que le type de traitement employé. Le disque Clair de terre, quatrième recueil de ses œuvres chez Empreintes Digitales, est probablement aussi son opus le plus personnel. La pièce titre, de 1999, fait référence à une série de concerts que produisait le compositeur entre 1989 à 1993 au Planétarium de Montréal, alors qu'il était membre de l'Association pour la création ef la recherche électroacoustiques du Québec (ACREQ). L'œuvre est divisée en douze mouvements dont les titres sont empruntés au vocabulaire du cinéma ("Cadre étroit», "Mobilité des plans", "Montage rythmique", etc.). Normandeau, actif défenseur de l'expression "cinéma pour l'oreille " comme description de l'art acousmatique, dévoile ici son programme de façon transparente. Paradoxalement, ce terme qu'il revendique convient plus ou moins bien à son travail, car ce "cinéma" est beaucoup plus musical que "visuel ". En écoutant la pièce Clair de terre, certainement la plus acousmatique des trois présentées ici, les deux autres étant dérivées de musiques d'application, on arrive aisément à en imaginer une orchestration lavec un orchestre, tout de même, qui ferait aussi preuve d'imagination!).

On touche donc ici au "problème acousmatique ". Le compositeur Denis Dufour a remplacé le mot "musique» par le mot "art», fatigué qu'il était de devoir sans cesse expliquer en quoi une pièce acousmatique pouvait se rapprocher ou s'éloigner de la musique ${ }^{\prime 2}$. C'est qu'en effet, l'art acousmatique ne produit pas toujours de la musique el certaines ceuvres ont certes plus à voir avec le cinéma ou le documentaire radiophonique. Ce n'est pas le cas ici et il serait inutile de bouder notre plaisir pour des considérations idéologiques.

Le disque s'ouvre sur Malina (2000), œuvre issue de la musique composée pour l'adaptation théâtrale du roman éponyme d'Ingeborg Bachmann. Le seul matériau ayant servi à la construction de la pièce est un enregistrement de la flûtiste Claire Marchand jouant du shakuhachi, une flûte japonaise. Évidemment, cet enregistrement est passé par toutes les transformations que peut lui faire subir un électroacousticien et notre petite flûte japonaise traditionnelle acquiert des qualités qui n'ont jamais été les siennes, se métamorphosant en instrument de percussion, puis en orgue ou en harpe éolienne. Le compositeur y crée davantage des ambiances qu'il n'y dépeint des paysages. Une grande réussite musicale suivie d'une autre, avec la pièce la plus récente du disque, Erinyes (2001), autre musique pour le théâtre basée sur l'emploi d'un seul matériau, en l'occurrence la voix. Cette façon de construire une œuvre à partir d'un matériau unique en le transformant de mille manières montre bien les prodigieuses capacités du médium électroacoustique. II ne pourrait que s'agir d'un exercice pédagogique, mais le talent du compositeur en fait tout autre chose. Enfin, Clair de terre, longue suite de trente-six minutes, nous présente un hommage à la musique concrète, cette musique qui se monte en studio et
12. Voir à ce sujet l'excellent entretien réalisé par Jonathan Prager avec Denis Dufour et publié dans les nos 5 (avril 1997), 6 (septembre 1997) et 7 (mars 1998) de la revue électronique Ars Sonora (hitp://ipt.univ-paris8.fr/ arsonora/). 
se projette en salle, comme le cinéma, selon l'expression de François Bayle. Les nouvelles façons d'appréhender la vie qu'ont pu provoquer les premières images de la Terre vue de l'espace avaient été précédées par les nouvelles façons de concevoir le son que proposait la musique concrète. C'est la vision d'un monde qui change et, c'est bien connu, en acousmatique, on voit mieux les yeux fermés. Évidemment, le thème de l'œuvre appelle l'utilisation de sons aux textures évocatrices mais de ces bruits, Normandeau forge un poème symphonique qui ne se prive pas $d$ 'avoir par moments recours à la pulsation et qui se termine même, tel un salut solennel au XX siècle, sur un air de cornemuse.

Surprise à la parution du disque Humeur de facteur d'Yves Beaupré. C'est que le compositeur n'est pas une figure connue du monde de la musique électroacoustique, mais plutôt des cercles de musique baroque, où on louange son habileté à... fabriquer des clavecins... Suivant un parcours peu orthodoxe pour un compositeur de musique électroacoustique, Beaupré se consacre en effet à la fabrication de clavecins, épinettes et virginals depuis une vingtaine $d^{\prime} a_{n n e ́ e s}{ }^{13}$, activité à laquelle il a commencé à s'intéresser au sortir d'études en interprétation clavecin à l'Université de Montréal. Évidemment préoccupé par la sonorité des instruments qu'il conçoit, le facteur a voulu en superviser la prise de son lors de séances d'enregistrement et a développé naturellement une passion pour la manipulation des objets sonores. Sa note biographique ne spécifiant pas d'études en composition, nous en déduisons qu'il est sans doute autodidacte. Les matériaux de base utilisés ici ont été enregistrés dans l'atelier du facteur et les multiples outils qu'il doit utiliser fournissent au preneur de son une matière riche.

Si les références au cinéma sont monnaie courante lorsqu'il est question de ce type de musique, Beaupré, lui, préfère évidemment une autre métaphore. La construction d'une pièce de ce type exige un travail tout aussi précis que celle d'un clavecin; dans les deux cas, les éléments bruts doivent "être manipulés, triturés, $[. .$.$] coupés, recoupés dans tous les sens { }^{14}$ » avant de seulement approcher le produit fini. C'est Beaupré l'interprète qui a influencé la forme sous laquelle se présente Humeur de facteur, calquée sur le modèle d'organisation des livres pour le clavecin de François Couperin, divisés en "ordres " qui contiennent eux-mêmes un nombre variable de pièces. On a donc ici un ordre dans lequel "chaque pièce, malgré son unicité, est partie intégrante du tout ${ }^{15}$ ". Les titres choisis pour les pièces sont certes moins évocateurs que ceux de Couperin, qui les nommait La majestueuse, la ténébreuse ou la lutine. On a ici la beauséjour, la pelots point ou la $D D B$, titres qui ne s'accompagnent pas d'explications, à l'auditeur donc de leur donner un sens.
13. Le 3 mai 2002 , le claveciniste luc Beauséjour présentait le $100^{\circ}$ instrument de Beaupré, un clavicythérium, à la Chapelle Notre-Dame-de-Bonsecours de Montréal.

14. Extrait de la notice du livret.

15. Idid. 
Le disque que présente Yves Beaupré a quelque chose de rafraîchissant, qui n'est probablement pas étranger à une certaine inexpérience de la part du compositeur, qui dote à l'ensemble d'une esthétique très personnelle.

Empreintes Digitales complète son catalogue 2001 en publiant des travaux récents du doyen des électroacousticiens québécois. Le disque de Francis Dhomont Cycle du son ${ }^{16}$, ajoute à cette année diversifiée un regard sur le médium lui-même, de sa naissance à ses développements les plus récents.

L'œuvre, en quatre volets, date de 1998, année qui marqua le cinquantième anniversaire de l'«invention du son» par Pierre Schaeffer au Studio d'essai de la RTF. Dhomont emprunte d'ailleurs à ce dernier, ainsi qu'à de nombreux autres "inventeurs du trésor», comme il les nomme, une partie du matériel sonore utilisé. Les Objets retrouvés (1996) sont un hommage direct à Schaeffer, en forme de paraphrase à son Étude aux objets. AvarArsSon (1998), véritable Who's Who de la musique concrète est un coup de chapeau aux Bayle, Chion, Henry, Parmegiani, Stockhausen, etc. Novars (1989) met en relation la "musique nouvelle" de Schaeffer et l'Ars Nova de Guillaume de Machaut et, enfin, Phonurgie (1998) annonce la poursuite de l'aventure, avec de nouveaux moyens, certes, mais en demeurant fidèle "à l'esprit des premiers concerts de bruits ${ }^{17}$ ".

Francis Dhomont a acquis une telle maîtrise du langage de la musique concrète que ce Cycle du son prend l'allure d'une véritable fête pour l'oreille. Et si chacun des volets est une réussite en soi, le tout est effectivement bien plus grand que la somme de ses parties. C'est que, comme le prouvaient déjà nombre de ses œuvres précédentes (du Cycle de l'errance à la Frankenstein Symphony), Dhomont est extrêmement à l'aise dans la grande forme. L'unité du matériau de base, assurée par l'utilisation des emprunts à Schaeffer ef les retours/rappels qui se font d'une œuvre à l'autre, permettent à l'auditeur de suivre le chemin tracé par le compositeur... les yeux fermés.

Vibrant hommage aux collègues, le Cycle du son est aussi un splendide témoignage de l'amour du métier par l'un de ses plus dignes représentants. Non pas un regard embué par la nostalgie, mais plutôt une fière mesure du chemin parcouru, résumé pas quelqu'un qui va droit au but.
16. La sartie de ce disque fut retardée à cause d'un problème d'impression de l'emballage. Profitons de l'occasion pour dire quelques mots sur la présentation de ces enregistrements. l'emballage a beaucoup évolué depuis les premières productions de l'étiquette, allant du modèle standard de boîtier en plastique à ces nouveaux boîtiers « Opaks * tout en carton. La nécessité étant mère de l'invention, c'est pour éviter le bris des boitiers standards lors d'envois postaux que ces mutations se sont opérées. La présentation visuelle est très soignée et les notes de programme, si leur consultation est moins pratique que celle du livret habituel, sont en général assez complètes.

17. Extrait de la notice du livret. 
照等 